\title{
Differences in the Isokinetic Strength of Thigh Muscles between Track and Field and Karate Athletes
}

\author{
Amel Mekic ${ }^{1}$, Safet Kapo', Haris Alic', Izet Bajramovic ${ }^{1}$, Slavenko Likic', Damir Besirevic ${ }^{1}$ and Nedim Covic ${ }^{1}$ \\ ${ }^{1}$ University of Sarajevo, Faculty of Sport and Physical Education, Sarajevo, Bosnia and Herzegovina
}

\begin{abstract}
This research aimed to determine the isokinetic strength differences between two groups of athletes (karate and track-and-field athletics) and to analyse factors that possibly contribute to the differences. We examined possible differences in peak power output and power ratio between agonist and antagonist thigh muscles of the knee. The sample consisted of 20 respondents: karate athletes $(n=10$; age 19 \pm 2.4$)$ and track-and-field athletes $(n=10$; age $18 \pm 2.6)$. For this study, a valid test $(\mathrm{CV}<5 \%)$ of the isokinetic strength output of the knee extensors and flexors, was used at the angular velocity of $60 \%$ s. Isokinetic variables: Peak torque in extension for both legs (Nm); Peak torque in flexion for both legs $(\mathrm{Nm})$; Total work for both legs $(\mathrm{J})$; Strength deficit involved/uninvolved leg and agonist/antagonist ratio for involved and uninvolved leg. A t-test for independent samples was used to determine the differences. Statistical significance was set at the conventional 95\%. In the sample of examined variables, the first tested group of athletes (karate) achieved higher power output values with the dominant leg, except in the case of the peak torque extensors. In the second tested group of athletes (track-and-field athletics), a higher power output values are registered with dominant leg, except in the case of the total work flexor with almost identical value of dominant and non-dominant leg. In the variables of the peak torque of the dominant leg $(p=0.002)$ and the peak torque of the non-dominant leg $(p=0.019)$, statistically significant differences were noted between two tested groups of athletes $(p<0.01, p<0.05)$. The unilateral relationship of the dominant leg $(p=.003)$ significantly differentiates two groups of athletes $(p<0.01)$. The better performance of track-and-field athletes is probably the result of the specificity of the structure of their motor movement and greater muscular work in training and competition. In contrast, the lower results of peak torque and total work in karate athletes compared to track athletes do not necessarily mean situational inferiority. Evaluation and assessment of knee dynamic stabilizers' isokinetic profile can lead to the optimal selection of training operators, during the construction of the overall training program for athletes. Results of different outputs of force and strength may indicate a differently shaped approach to training.
\end{abstract}

Keywords: knee dynamic stabilizers, flexor and extensor, training specificity, evaluation

\section{Introduction}

According to the International Association of Athletics Federations (IAAF), the athletics track disciplines are sprint (100 m, $200 \mathrm{~m}$ and $400 \mathrm{~m})$, middle and long distances (800 $\mathrm{m}, 1500 \mathrm{~m}, 5000 \mathrm{~m}, 10000 \mathrm{~m}$ and $3000 \mathrm{~m}$ steeplechase); hurdles (men: $110 \mathrm{~m}$ and $400 \mathrm{~m}$; 100 women $100 \mathrm{~m}$ and 400 $\mathrm{m}$ ) and road running (marathon and half-marathon), as well as Relay (4x100 m; 4x400 m); cross country running; mountain running and ultra-running disciplines. Sprint disciplines and sprinting ability is based on lower extrem-

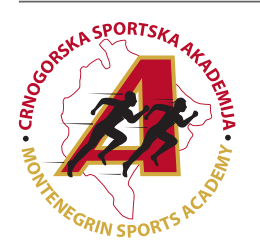

Correspondence:

S. Likic

University of Sarajevo, Faculty for Sport and Physical Education, Patriotske lige 41, 71000 Sarajevo, Bosnia and Herzegovina E-mail: slikic@fasto.unsa.ba 
ities strength capacity. The long-term training process can initiate different effects in leg strength balance, as well as the unilateral and bilateral strength ratio. Sprinters are faster at $20 \mathrm{~m}$ and have more speed at block when their take-off leg is dominant (Vagenas \& Hoshizaki, 1986). Asymmetry of dynamic leg strength is key factor for successful start (Vagenas \& Hoshizaki, 1986; Exell, Irwin, Gittoes, \& Kerwin, 2017). Most of the strength asymmetry is registered in the ankle and upper leg muscles (Exell et al., 2017); however, there is no correlation between leg strength asymmetry and running velocity. Also, because of the training structure and motor movements, sprint produces constant sudden shifting between maximal voluntary eccentric and concentric contraction, which significantly influences strength development, especially in hamstring muscles (Jonhagen, Nemeth, \& Eriksson, 1994).

Karate is different from other martial arts in that its basic goal, which is symbolic destruction of the opponent, is achieved by simulated or strictly controlled blows of the arms and legs (Sertić, 2004). Generally, punches account for $89.09 \%$ of the movements, leg kicks $8.36 \%$, while cleaning and throwing with pointing account for $2.55 \%$ (Koropanovski, Dopsaj, \& Jovanovic, 2008). The dynamics of movement in karate are particularly emphasized, while static situations most often occur when maintaining postures. Upper and lower body strength and maximum dynamic strength variables were positively correlated to punch acceleration in all conditions (Loturco, Artioli, Kobal, Gil, \& Franchini, 2014). Also, in karate, reaction time is a key element, because high performance is based on explosive techniques, while karate performance relies more on muscle strength at lower versus higher loads (Chaabène, Hachana, Franchini, Mkaouer, \& Chamari, 2012). Karate shows a specific sport adaptation when measuring power with an isokinetic dynamometer (Probst, Fletcher, \& Seelig, 2007).

The skeleton and joints are the dynamically inactive part while the muscles are the active part of the locomotors system, which ensures the movement of the body by its contractions. The symmetry of muscle strength for each joint and each direction of movement should be balanced so that the body remains upright or performs the desired movements. If the force-to-muscle ratio is exceeded, then injuries such as ligament or muscle fractures, cartilage damage in the joint, chronic inflammatory reactions, pain, and permanent joint damage occur (Hadžović-Džuvo \& Kapur, 2011). Greater asymmetries are noticeable in non-athletes (Siqueira, Pelegrini, Fontana, \& Greve, 2002).

Isokinetic testing provides detailed and exact insight into the strength of individual muscle groups, the ratio of muscle strength of agonists and antagonists, bilateral comparison of the same muscle groups, information on the amount of work performed, information on the moment of expression of maximum muscle strength during a particular motor task, and information on fatigue index (DesnicaBakrač, 2003). The aim of many previous studies was to evaluate muscle performance, primarily for the purpose of objectively documenting and reviewing the effectiveness of certain therapeutic procedures for the rehabilitation of injuries to the locomotors system, as well as for determining deficiencies in muscle strength and determining the relative strength of antagonistic muscle groups due to the influ- ence of dominance (Siqueira et al., 2002).

This research aimed to determine the isokinetic strength differences between two groups of athletes (karate and track-and-field athletics) and to analyse factors that possibly contribute to those differences. I

\section{Methods}

Inclusion criteria featured male athletes 16-21 years of age. For this study, a valid test $(\mathrm{CV}<5 \%)$ of the isokinetic strength output of the knee extensor's and flexor's, was used at the angular velocity of $60^{\circ} \%$ s. Age and morphological characteristics (height and body mass) of the first tested group of athletes (karate): $\mathrm{n}=10,19 \pm 2.4,184 \pm 7 \mathrm{~cm}, 77.8 \pm 11.5 \mathrm{~kg}$; and second tested group of athletes (track-and-field athletics): $\mathrm{n}=10,18 \pm 2.6,182 \pm 5.4 \mathrm{~cm}, 76.1 \pm 4.5 \mathrm{~kg}$.

Isokinetic variables (Biodex System): Peak torque dominant leg Extensor (Nm), Peak torque non-dominant leg Extensor $(\mathrm{Nm})$, Peak torque dominant leg Flexor $(\mathrm{Nm})$, Peak torque non-dominant leg Flexor (Nm), Total work dominant Extensor (J), Total work non-dominant Extensor $(\mathrm{J})$, Total work dominant Flexor (J), Total work non-dominant Flexor (J), Unilateral ratio dominant leg, Unilateral ratio non-dominant leg.

A t-test for independent samples was used to determine the differences. Statistical significance was set at the conventional 95\%.

All subjects were completely healthy, with no recorded knee joint injuries, and at their discretion participated in the study and isokinetic testing procedure. All tested athletes did this isokinetic testing from 2012 to 2017 . The measurement protocol involved a standard warm-up procedure after which the subject performed five maximal voluntary contractions at an angular velocity of $60^{\circ} \%$ s. An $80^{\circ}$ range of motion was set for each subject. The rotation axis of the knee joint is aligned with the axis of rotation of the isokinetic dynamometer. The gravity correction was performed at an angle of $30^{\circ}$ with respect to the vertically positioned isokinetic dynamometer. Each subject was in a specific position: sitting on an isokinetic chair.

Statistical programs SPSS and EXCEL were used for data processing. All result values are presented as arithmetic mean and standard deviation. Differences in outcome values between the two groups of athletes were determined using the two-sided t-test. Statistical significance was set at the conventional $95 \%$.

\section{Results}

The karate athletes achieved better results with their dominant leg in all variables, except for the Peak torque Extensor variable which indicates a better result achieved with the non-dominant leg (Table 1). The skewness values indicate that all variables have a positive sign, of which seven results have slightly more pronounced asymmetry values. This means that most of the results are poorly grouped; that is, there are one or several extremely high values in karate athletes. The total work non-dominant Flexor and Unilateral ratio dominant leg variables are closest to ideal symmetry. The values of kurtosis indicate that most of the results in karate athletes have a mesokurtic distribution, and the closest to the ideal distribution is variables Peak Torque dominant leg Extensor and Total work dominant Extensor. The tendency toward platykurtic distribution is noticeable 
in the variable Unilateral ratio dominant leg. Variables that analyse the unilateral ratio of power output between flexors and extensors, based on the values of arithmetic means, may suggest a better-quality relationship with the dominant leg.

Table 1. Descriptive isokinetic parameters of karate athletes

\begin{tabular}{lccccc}
\hline \multicolumn{1}{c}{ Variables } & Minimum & Maximum & Mean \pm SD & Skewness & Kurtosis \\
\hline Peak torque dominant leg Extensor & 202.10 & 289.30 & $230.91 \pm 28.88$ & .93 & .16 \\
Peak torque non-dominant leg Extensor & 199.60 & 309.90 & $236.10 \pm 35.34$ & .90 & .62 \\
Peak torque dominant leg flexor & 114.50 & 180.10 & $138.13 \pm 21.44$ & 1.03 & .40 \\
Peak torque non - dominant leg flexor & 99.40 & 180.60 & $129.15 \pm 24.56$ & .86 & .97 \\
Total work dominant Extensor & 793.70 & 1362.10 & $995.33 \pm 187.18$ & .93 & .12 \\
Total work non - dominant Extensor & 763.10 & 1231.90 & $968.35 \pm 143.95$ & .60 & -.32 \\
Total work dominant Flexor & 491.10 & 878.50 & $637.17 \pm 139.69$ & .97 & -.36 \\
Total work non-dominant Flexor & 437.40 & 734.60 & $582.55 \pm 97.72$ & .13 & -.94 \\
Unilateral ratio dominant leg & 55.40 & 64.90 & $59.71 \pm 3.79$ & .18 & -1.91 \\
Unilateral ratio non-dominant leg & 48.90 & 63.90 & $54.58 \pm 4.91$ & .46 & -.37 \\
\hline
\end{tabular}

Track-and-field athletes also achieved better results with their dominant leg (Table 2). The only exception is the variable Total work dominant Flexor, in which a nearly identical result was recorded with dominant and non-dominant leg. Regardless of the positive or negative sign, skewness values indicate that all but one result is within the allowed distribution. An exception is the extreme negative asymmetry of results in the Peak torque dominant leg Extensor variable in track-andfield athletes. This suggests grouping better results than the arithmetic mean, that is, the presence of one or several ex- tremely lower values. Peak torque non-dominant leg Extensor and Unilateral ratio non-dominant leg are close to ideal symmetry of 0 . Most kurtosis results indicate mesokurtic or a slight platykurtic tendency. The result of the Peak torque dominant leg Extensor suggests a leptokurtic distribution of results, that is, the accumulation of most results around the centre of distribution. The unilateral ratio of power output between flexors and extensors in track-and-field athletes, based on the values of arithmetic means, suggest an equal ratio for both legs, which is lower than the recommended value of 61 (Biodex System).

Table 2. Descriptive isokinetic parameters of track-and-field athletes

\begin{tabular}{lccccc}
\hline \multicolumn{1}{c}{ Variables } & Minimum & Maximum & Mean \pm SD & Skewness & Kurtosis \\
\hline Peak torque dominant leg Extensor & 197.10 & 330.60 & $283.91 \pm 35.80$ & -1.59 & 3.95 \\
Peak torque non-dominant leg Extensor & 211.70 & 343.80 & $280.45 \pm 41.62$ & .05 & -.92 \\
Peak torque dominant leg flexor & 113.60 & 169.70 & $148.07 \pm 19.03$ & -.52 & -.91 \\
Peak torque non-dominant leg flexor & 112.30 & 166.50 & $143.68 \pm 19.57$ & -.31 & -1.10 \\
Total work dominant Extensor & 892.90 & 1354.30 & $1142.37 \pm 139.69$ & -.21 & -.45 \\
Total work non-dominant Extensor & 936.50 & 1347.00 & $1092.06 \pm 127.56$ & .87 & .18 \\
Total work dominant Flexor & 533.50 & 744.40 & $652.48 \pm 74.02$ & -.60 & -.87 \\
Total work non-dominant Flexor & 521.80 & 830.10 & $652.65 \pm 102.74$ & .34 & -1.00 \\
Unilateral ratio dominant leg & 45.30 & 60.40 & $52.43 \pm 5.56$ & .18 & -1.47 \\
Unilateral ratio non-dominant leg & 40.60 & 64.40 & $52.01 \pm 7.88$ & .07 & -.75 \\
\hline
\end{tabular}

Comparing the result of arithmetic means between two tested groups of athletes, it is evident that the variable Peak torque dominant leg Extensor in karate athletes has a lower value by $53 \mathrm{Nm}$ compared to the value measured in trackand-field athletes, or by $44.4 \mathrm{Nm}$ in the variable Peak torque non-dominant leg Extensor. The peak torque dominant leg Flexor is slightly higher for track-and-field athletes by $9.9 \mathrm{Nm}$ or $14.5 \mathrm{Nm}$ for Peak torque non-dominant leg Flexor. The total work of dominant and non-dominant legs in extensors is higher in track-and-field athletes than in karate athletes (147 Nm and $123.7 \mathrm{Nm}$ ). The total work of the dominant and non-dominant legs in flexors measured in track-and-field athletes is also higher than measured values of karate athletes (15.3 Nm and 70.1 Nm). The unilateral ratio of the dominant leg is higher for track-and-field athletes compared to karate athletes by $7.3 \mathrm{Nm}$, and by $2.6 \mathrm{Nm}$ in the case of the unilateral ratio of non-dominant leg.

Statistically significant differences between the two tested groups of athletes are evident in the following variables (Table 3): Peak torque dominant leg Extensor $(\mathrm{p}=0.002)$, Peak torque non-dominant leg Extensor $(\mathrm{p}=0.019)$ and Unilateral ratio dominant leg $(p=.003)$. A negative sign of the $t$-test in the variables Peak torque dominant leg Extensor and Peak torque non-dominant leg Extensor indicates a better power output values of the track-and-field athletes. On the other hand, a positive sign of the t-test in the variable Unilateral ratio dominant leg indicates a better power output ratio in the case of karate athletes. 
Table 3. Independent t-test results between karate and track-and-field athletes

\begin{tabular}{lcll}
\hline \multicolumn{1}{c}{ Variables } & $\mathbf{t}$ & Df & Sig. \\
\hline Peak torque dominant leg Extensor & -3.644 & 18 & $.002^{*}$ \\
Peak torque non-dominant leg Extensor & -2.569 & 18 & $.019^{*}$ \\
Peak torque dominant leg flexor & -1.096 & 18 & .287 \\
Peak torque non-dominant leg flexor & -1.463 & 18 & .161 \\
Total work dominant Extensor & -1.991 & 18 & .062 \\
Total work non-dominant Extensor & -2.034 & 18 & .057 \\
Total work dominant Flexor & -.306 & 18 & .763 \\
Total work non-dominant Flexor & -1.563 & 18 & .135 \\
Unilateral ratio dominant leg & 3.423 & 18 & $.003^{*}$ \\
Unilateral ratio non-dominant leg & .875 & 18 & .393 \\
\hline
\end{tabular}

Legend: ${ }^{*}-p<0.05$

\section{Discussion}

By analysing the track and field athlete's training structure, it is possible to assume the reason for their dominance in relation to karate athletes. Track-and-field athletes' training is characterized by various motor movements that develop the entire musculature. The specificity of training and competitions in karate is likely to make more impact with a dominant leg. The dominance of one leg may cause asymmetry between muscle groups and develop a predisposition for injury (Zakas, 2006). Different resistance training programs should consider the specific neuromuscular demands of each sport (F.B.D. Oliveira, A.S.C. Oliveira, Rizatto, \& Denadai, 2013). Training programs are linked to different athlete profiles, models and requirements of a particular sport, which requires the correct selection of exercises. The load distribution of exercises needs to be carefully selected, depending on the sport as well as the individual in the sport.

The results obtained with the isokinetic protocol in the treated groups of karate and track and field athletes are within the optimal values. Track and field athlete's better test-results are probably the result of the specificity of the structure of their motor movement and greater muscular work in training and competition. In track and field athlete's training, the mechanical work of the lower extremities is particularly pronounced, which involves performing high-speed and explosive movements, as well as very high engagement of the muscles of the upper leg (m. quadriceps femoris, $\mathrm{m}$. biceps femoris, $\mathrm{m}$. semitendinosus and $\mathrm{m}$. semimembranosus). This is especially pronounced in the track and field sprint disciplines. Greater muscular strength is strongly associated with improved force-time characteristics that contribute to an athlete's overall performance (Suchomel, Nimphius, \& Stone, 2016). The results of this study indicate the presence of a low tendency for muscular asymmetry on both legs in track-and-field athletes. Similar results were obtained in the study of Siqueira et al. (2002). The training of track-and-field athletes abound in various movements, like sprints, jumps, and throws that optimally develop the muscles of the entire body. Specifically, concentric and eccentric contraction predominates in muscles at high levels of dynamic loading, whereby activities are performed under conditions of overcoming and ease up force muscle work. The result of such high-intensity loads is an improvement in the increase in muscle force and muscle strength. In contrast, it is necessary to make an optimal distribution of training load in order to avoid harmful consequences.
Karate is full of various motor movements, such as walking, running, sprints, jumps, changes of movement direction, and duels. The decisive actions of karate depend essentially on the explosive power of the muscles in the upper and lower extremities (Chaabène et al., 2012). However, karate athletes achieve better results with their dominant leg, probably because of its more frequent use in training and competition. Andrzejewski and Elbaum (2005) state that, in karate, the kinematics of impact with non-dominant extremities is quite similar to the dominant side, but lower angular and linear impact velocities have also been reported. Karate training can produce agonist-antagonistic muscle asymmetries that can predispose these athletes to knee joint injuries (Scattone-Silva, Lessi, Lobato, \& Serrão, 2012). Kovač, Kovačević, Abazović, and Alić (2013) emphasize the importance of preventive training primarily aimed at improving power, strength and muscular endurance, and reducing bilateral and reciprocal muscle group deficits.

However, lower values in Peak torque and Total work of karate athletes in relation to track-and-field athletes do not necessarily mean their situational inferiority. The treated groups of athletes, according to the periodization of sports development, belong to the stage of adolescence, and their performance is close to elite sports performance. However, chronological age is not the most appropriate method for analysing biological data in adolescents (Bjelica, 2013). Creating a training program, in contrast, is directly linked to the athlete profiles, models and requirements of a particular sport. This indicates in practice that the selection of exercises and the distribution of exercise load should be carefully chosen in relation to sport and also in accordance with the individual approach to athletes. The evaluation of the isokinetic profile of dynamic knee stabilizers can guide the optimal selection of training operators as well as the creation of the overall athlete training program. Results on different outputs of muscle force and muscle strength may indicate a differently shaped approach to training. In addition to isokinetic testing procedures, the use of basic and specific tests is the first recommendation, both in karate and track-and-field athletics practice. Also, the disadvantage of isokinetic measurement is its realization under open kinetic chain conditions, whereas in real sports situations, the moments of the open and closed kinetic chain are most often exchanged. Hence, functional training is a form of the fastest and most effective action to increase strength and build muscles (Thomee, Augustsson, Wernbom, Augustsson, \& Karlsson, 2008). 
Acknowledgements

There are no acknowledgements.

\section{Conflict of Interest}

The authors declare that there are no conflicts of interest.

Received: 09 September 2019 | Accepted: 30 October 2019 | Published: 01 February 2020

\section{References}

Andrzejewski, X., \& Elbaum, L. (2005). Biomechanical analysis of the front kick with the dominant and non-dominant limb in the shito-rvu style of karate. 23nd International Symposium on Biomechanics in Sports (22-27), China: Beijing.

Bjelica, D. (2013). Theory of sports training. Podgorica, Montenegro: University of Montenegro.

Chaabène, C., Hachana, Y., Franchini, E., Mkaouer, B., \& Chamari, K. (2012). Physical and Physiological Profile of Elite Karate Athletes. Sports Medicine, 42(10), 829-843.

Desnica-Bakrač, N. (2003). Isokinetic diagnostics. Conditioning training, 1(2), 7-13.

Exell, T., Irwin, G., Gittoes, M., \& Kerwin, D. (2017). Strength and performance asymmetry during maximal velocity sprint running. Scandinavian journal of medicine \& science in sports, 27(11), 1273-1282.

Hadžović-Džuvo, A., \& Kapur, E. (2011). Basics of human anatomy and physiology with sports physiology. Sarajevo, Bosnia and Herzegovina: University of Sarajevo - Faculty of Sport and Physical Education.

Jonhagen, S., Nemeth, G., \& Eriksson, E. (1994). Hamstring injuries in sprinters: the role of concentric and eccentric hamstring muscle strength and flexibility. The American Journal of Sports Medicine, 22(2), 262-266.

Loturco, I., Artioli, G.G., Kobal, R., Gil, S., \& Franchini, E. (2014). Predicting punching acceleration from selected strength and power variables in elite karate athletes: A multiple regression analysis. J Strength Cond Res 28(7), 1826-1832. doi: 10.1519/JSC.0000000000000329

Koropanovski, N., Dopsaj, M., \& Jovanovic, S. (2008). Characteristics of point- ing actions of top male competitors in karate at world and European level. Brazilian Journal of Biomotricity, 2(4), 241-251.

Kovač, S., Kovačević, E., Abazović, E., \& Alić. H., (2013). Isokinetic testing and training. Sarajevo, Bosnia and Herzegovina: University of Sarajevo - Faculty of Sport and Physical Education.

Oliveira, F.B.D., Oliveira, A.S.C., Rizatto, G.F., \& Denadai, B.S. (2013). Resistance Training for Explosive and Maximal Strength: Effects on Early and Late Rate of Force. J Sports Sci Med., 12(3), 402-408.

Probst, M., Fletcher, R., \& Seelig, D.S. (2007). A Comparison of Lower-Body Flexibility, Strength, and Knee Stability between Karate Athletes and Active Controls. Journal of Strength and Conditioning Research, Champaign, 21(2), 451-5.

Scattone-Silva, R., Lessi, G.C., Lobato, D.F.M., \& Serrão, F.V. (2012). Acceleration time, peak torque and time to peak torque in elite karate athletes. Science \& Sports, 27(24), 31-37. https://doi.org/10.1016/j.scispo.2011.08.005

Sertić, H. (2004). Osnove borilačkih vještina. Zagreb, Croatia: Kineziološki fakultet Sveučilišta u Zagrebu.

Siqueira, C.M., Pelegrini, F.R.M.M., Fontana, M.F., \& Greve, J.M.D. (2002). Isokinetic dynamometry of knee flexors and extensors: comparative study among non-athletes, jumper athletes and runner athletes. Rev. Hosp. Clin., 57(1), 19-24. http://dx.doi.org/10.1590/S004187812002000100004

Suchomel, T., Nimphius, S., \& Stone, M. (2016). The Importance of Muscular Strength in Athletic Performance. Sports Medicine, 46(10), 1419-49. https://doi.org/10.1007/s40279-016-0486-0

Thomee, R., Augustsson J., Wernbom, M., Augustsson, S., \& Karlsson J. (2008). Strength training for sports, exercise and rehabilitation. Stockholm: SISU Idrottsböcker.

Vagenas, G., \& Hoshizaki, T. B. (1986). Optimization of an asymmetrical motor skill: Sprint start. Journal of Applied Biomechanics, 2(1), 29-40.

Zakas, A. (2006). Bilateral isokinetic peak torque of quadriceps and hamstring muscles in professional soccer players with dominance on one or both two sides. J Sports Med Phys Fitness, 46(1), 28-35. 